\title{
Avalanche photo diodes in the observatory environment: lucky imaging at 1-2.5 microns
}

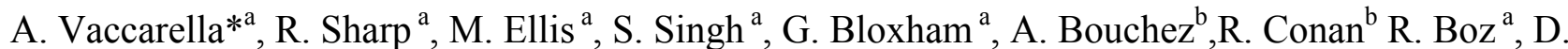

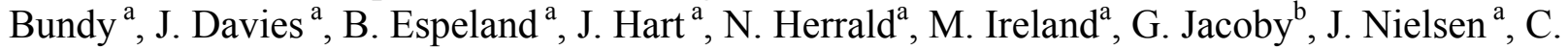 \\ Vest $^{\mathrm{a}}$, P. Young ${ }^{\mathrm{a}}$, B. Fordham ${ }^{\mathrm{a}}$, A. Zovaro ${ }^{\mathrm{a}}$ \\ ${ }^{a}$ Australian National University, Mt Stromlo Observatory, Weston ACT, Australia, 2611 \\ ${ }^{\mathrm{b}}$ GMTO Corporation, 465 N. Halstead St, Suite 250, Pasadena, CA, 91107, USA.
}

\begin{abstract}
The recent availability of large format near-infrared detectors with sub-election readout noise is revolutionizing our approach to wavefront sensing for adaptive optics. However, as with all near-infrared detector technologies, challenges exist in moving from the comfort of the laboratory test-bench into the harsh reality of the observatory environment. As part of the broader adaptive optics program for the GMT, we are developing a near-infrared Lucky Imaging camera for operational deployment at the ANU $2.3 \mathrm{~m}$ telescope at Siding Spring Observatory. The system provides an ideal test-bed for the rapidly evolving Selex/SAPHIRA eAPD technology while providing scientific imaging at angular resolution rivalling the Hubble Space Telescope at wavelengths $\lambda=1.3-2.5 \mu \mathrm{m}$.
\end{abstract}

Keywords: eAPD, wavefront sensing, near-infrared, lucky imaging.

\section{INTRODUCTION}

As part of ongoing detector evaluation at the Australian National University (ANU), we are undertaking to deploy a Lucky Imaging instrument on the $2.3 \mathrm{~m}$ telescope at Siding Springs using a Selex SAPHIRA near-infrared electron Avalanche Photo-Diode (eAPD) array, capable of high cadence imaging with frame rates of $10-5,000 \mathrm{~Hz}$ over the wavelength range of $0.8 \mu \mathrm{m}$ to $2.5 \mu \mathrm{m}$. The controller for the initial development phase of the project will be an ARC SDSU Series III. A demountable cryostat for telescope operations will be developed in-house at ANU based on a design adaptation of the ANU/WiFeS instrument cryostat. ${ }^{2}$ A principle aim of this project is to develop in-house expertise with the emerging eAPD detector technology which is changing the paradigm for near-infrared wavefront sensing in astronomy.

In the first instance the instrument will be deployed on the ANU $2.3 \mathrm{~m}$ telescope at the Siding Spring Observatory. Simulations have shown that the instrument will be near diffraction limited with a field of view of $\approx 30$ " $\times 30$ " with image sampling at $0.12 "{ }^{6,7}$ The cryostat will be designed as a generic focal plane instrument suitable for high-cadence photometry and Lucky Imaging at other observatories.

In addition to demonstrating the science capability of the technology through the deployment of the Lucky Imaging instrument, delivering a prototype low readout-noise focal-plane wave-front sensor, essential for the Giant Magellan Telescope (GMT) adaptive optics (AO) and part of the GMTIFS instrument concept, ${ }^{3}$ will mitigate a major programmatic risk for the next generation of telescope facilities.

\section{READOUT ELECTRONICS}

The readout electronics will consist of a detector PCB carrying the Selex SAPHIRA APD and cryogenic preamplifiers interfacing to a 32 channel ARC SDSU III detector controller.

The venerable ARC SDSU III has been identified as the initial test controller due to our existing in-house experience with this devices (e.g., NIFS, GSAOI, WiFeS ${ }^{2,4,5}$ ); however it is envisaged that alternative controllers will be interfaced to the system in the future. The in-house expertise with programming the SDSU lends itself well to this project as it will

Ground-based and Airborne Instrumentation for Astronomy VI, edited by Christopher J. Evans, Luc Simard, Hideki Takami Proc. of SPIE Vol. 9908, 99082X · @ 2016 SPIE · CCC code: 0277-786X/16/\$18 · doi: 10.1117/12.2231557 
allow us to test the full suite of features available on the ME1000 readout integrated circuit (ROIC) allowing us to take full advantage of the new technology in future applications

To overcome the perennial problem of pickup noise in long control leads between cryogenic near-infrared detectors and external control electronics, we have developed a cryogenic preamplification circuit for use with the eAPD detector and the large format science arrays (e.g., the Teledyne HxRG detector series to be used with the GMTIGS instrument, Sharp et al. 2016). Indeed, the Lucky Imager program provides the perfect test bed for such developments.

Care has been taken to ensure that the circuits have been designed to minimise the noise in the readout chain. The ground planes are cut out around high impedance pins to avoid coupling between the signal pins and the ground plane. This is particularly significant for the input pins on the operational amplifiers on the cryogenic preamplifier circuit. The circuit boards all have internal ground planes to separate the analogue and digital signals as well as the differential and single ended signals. The clocks, biases and power signals are laid out over their own independent ground plane linked up to either the digital or analogue grounds. All differential pairs are balanced with equal length tracks and chamfered corners.

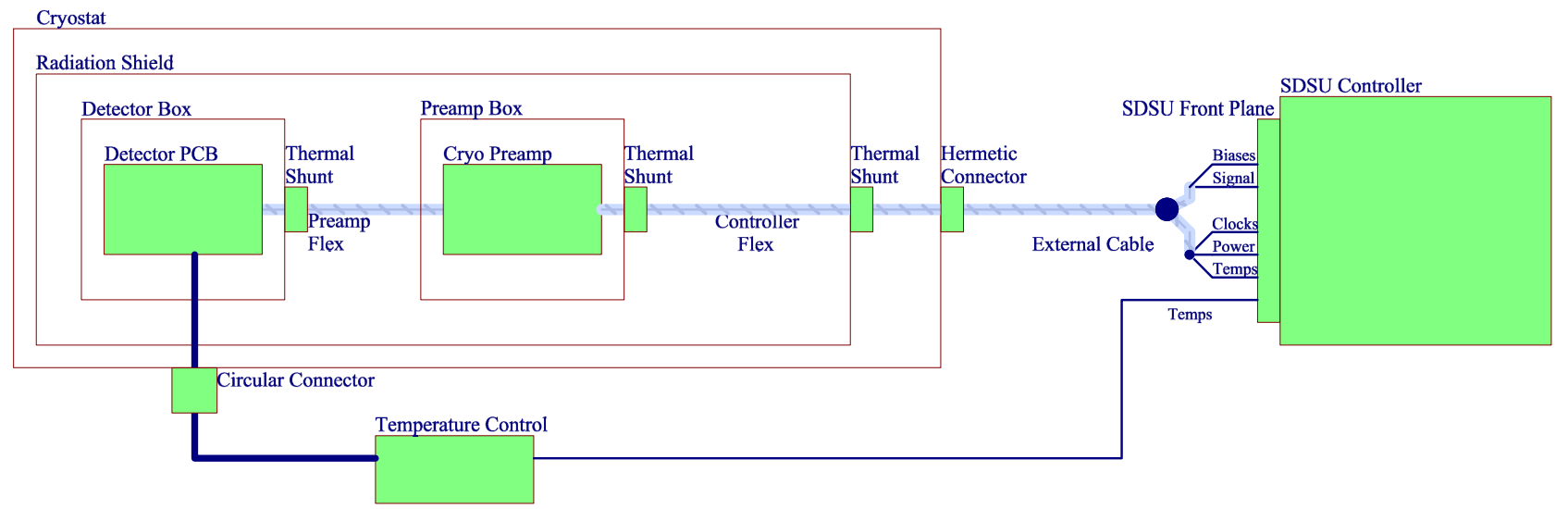

Figure 1. High level system overview for the ANU Lucky Imager.

\subsection{Detector PCB}

The detector PCB is primarily designed to carry the APD which is packaged in a 68-pin leadless package. However due to space constraints on the preamplifier circuit the bias filtering has been added to the underside of the detector PCB. The biases do not vary with increasing readout speeds, and so the heat produced by the filtering circuits will remain constant regardless of the readout speed. A middle ground layer in the PCB will be connected to the second stage cold head and cooled to the same temperature as the detector, which will provide additional radiation isolation between the filtering circuits and the detector. 


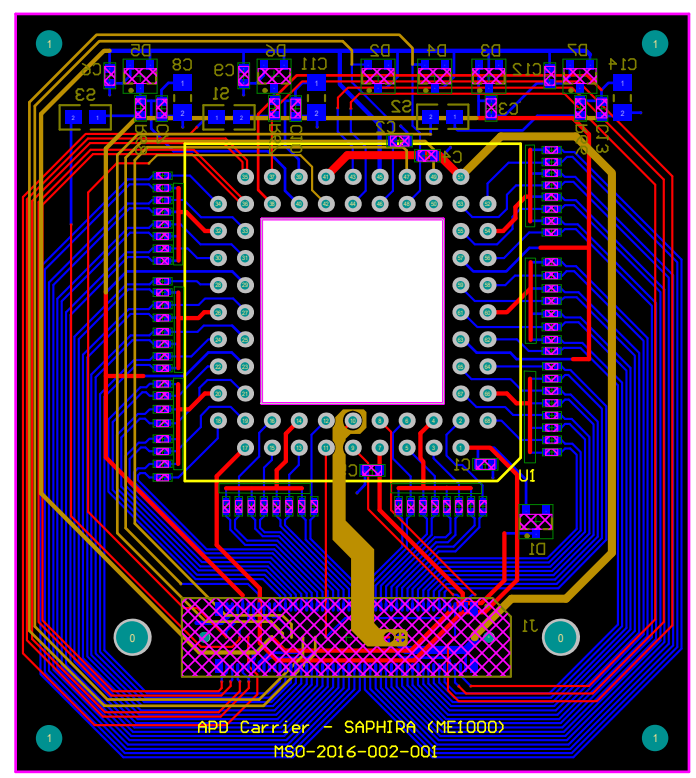

Figure 2. Detector PCB with the detector mounted on the top layer and all components for filtering mounted on the bottom layer. The open space in the center of the PLCC socket is for the detector cooling interface.

The APD will be cooled by a spring-loaded copper block in contact with the back of the Leadless Chip Carrier (LCC) package. The copper block will then be connected to the second stage cold head via two copper straps. To accommodate cooling, the SAPHIRA APD will be mounted to the detector PCB in an off-the-shelf 68-pin PLCC socket with the bottom cover removed. The PCB has a square cutout that matches the square cutout of the PLCC socket, as shown in Fig. 2.

The detector PCB and cooling interface will be enclosed within an aluminium box and cooled to the same temperature as the APD. The design for the detector PCB box has not been finalized, but the box could potentially act as an active radiation shield and photon shield.

The flex cable connecting the detector PCB to the preamplifier circuit will pass through a thermal shunt as it exits the detector box to isolate heat transfer between the two circuit boards. The flex cable has been kept to a length of $32 \mathrm{~mm}$. 


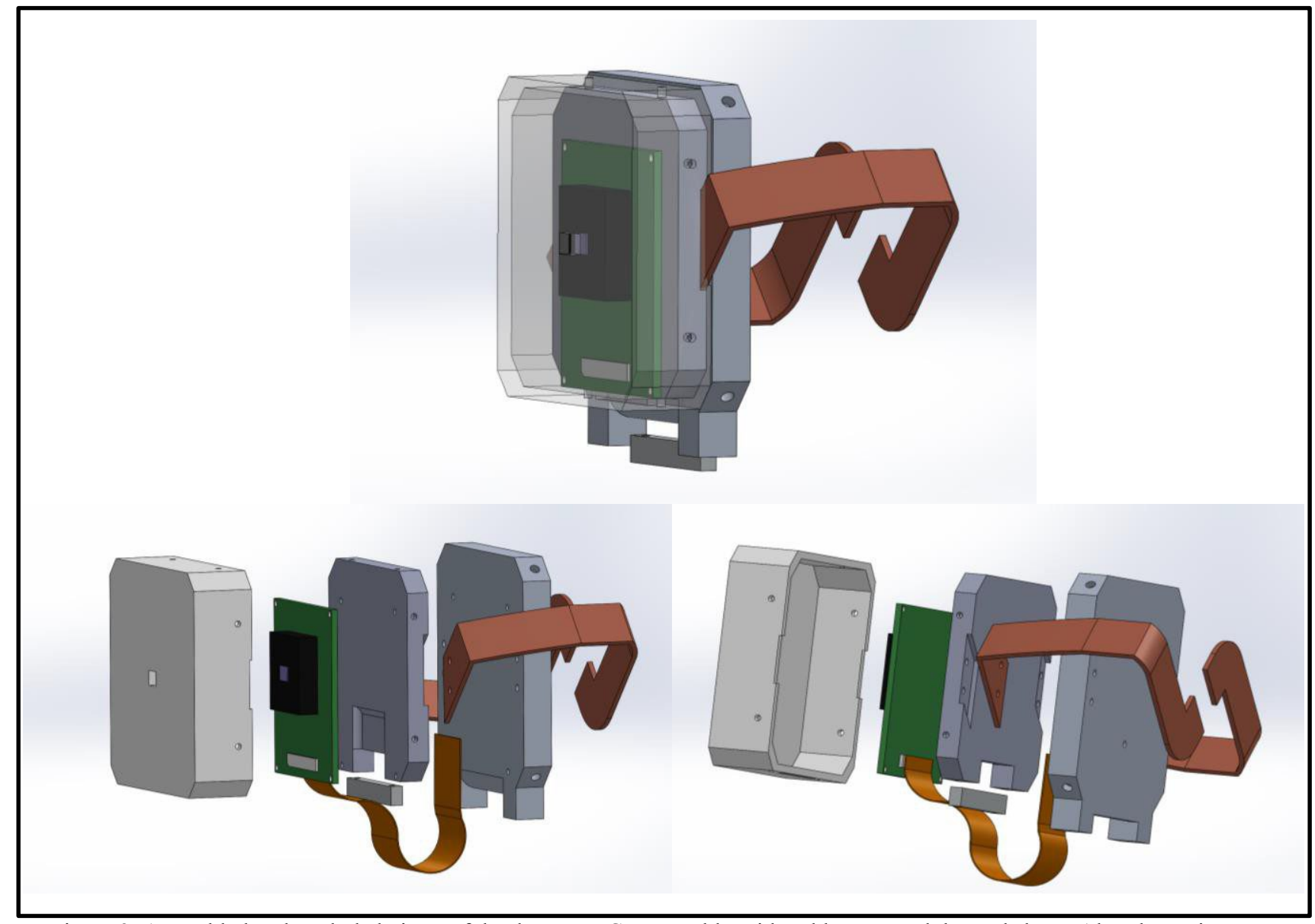

Figure 3. Assembled and exploded views of the detector PCB assembly with cold straps and thermal shunt. Also shown is the path the cold straps take around the cryogenic preamplifier box.

\subsection{Cryogenic Preamplifier}

The cryogenic preamplifier is a 32 channel instrument amplifier which is an extension of an existing 6 channel cryogenic preamplifier circuit designed and tested at the ANU to interface the Teledyne H1RG/H2RG detectors to the ARC SDSU III controller. The instrument amplifier circuit includes two OPA2354 op-amps which have proven to be very robust in cryogenic environments. The ANU has an existing detector mount design which can accommodate the Teledyne SIDECAR ASIC. To leverage the existing detector mount design, the cryogenic preamplifier circuit has been sized to occupy the same footprint as the Teledyne SIDECAR.

The cryogenic preamplifier circuit design has been tested at cryogenic temperatures as low as $50 \mathrm{~K}$ while interfaced to a Teledyne H2RG ROIC. During testing with the H2RG, the differential video signal from the preamplifier has proven to be robust against external interference.

The cryogenic preamplifier circuit is separated from the detector PCB to reduce the risk of glow from the op amps at the highest readout speeds. The cryogenic preamp circuit when operating at the highest frame rates will dissipate up to 340 $\mathrm{mW}$ of power. Maintaining the cryogenic preamp circuit at a higher temperature than the detector will reduce the load on the cooling system. The op-amps are actively cooled through the ground plane through the 4 mounting holes at each corner of the PCB. 


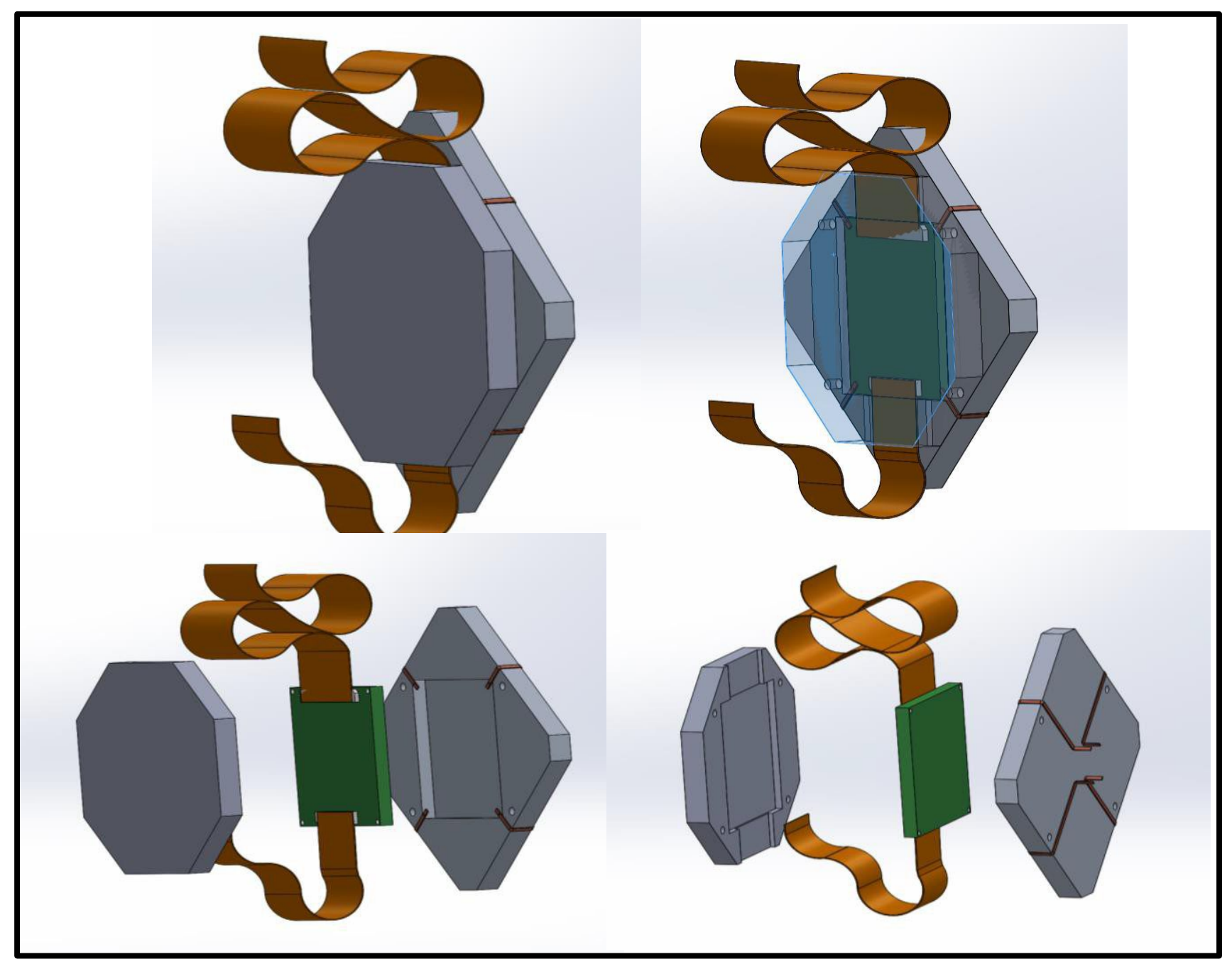

Figure 4. Exploded and blown up views of the cryogenic preamps assembly showing the four small copper straps for cooling the preamplifier PCB.

The cryogenic preamplifier and cooling interface will be enclosed within an aluminium box which will be cooled to the same temperature as the cryogenic preamplifier and could potentially act as an active radiation shield. The cryogenic preamplifier box has 4 small copper cold straps that will be screwed onto the top face of the preamplifier at the edges via holes. The cold straps will then go around the rear preamplifier box and join to a copper block which is directly connected to the cold head of the cryostat.

\subsection{Detector Controller}

The detector controller to be used initially will be a 32 channel ARC SDSU III. This controller has been chosen because it is easily available and we can leverage the considerable in-house knowledge to fully investigate and evaluate the features and performance of the latest Selex SAPHIRA with the ME1000 ROIC.

The SDSU controller does not have the capacity to push the SAPHIRA to the limits of its readout speed. The SDSU will only ever achieve a maximum frame rate of $100 \mathrm{~Hz},{ }^{1}$ but this will be sufficient to provide near-infrared images approaching the clarity of the Hubble Space Telescope at the ANU $2.3 \mathrm{~m}$ telescope at Siding Springs. The SDSU is also capable of reading out windowed regions of interest at the $1 \mathrm{kHz}$ rate, which will allow us to investigate the suitability of the SAPHIRA device for the GMT AO systems. 


\subsection{Temperature Interlock}

The APD array can become temporarily damaged when operated above $150 \mathrm{~K}$ at anything above minimal avalanche gain. This is thought to be caused by hot electron damage to the weak $\mathrm{Hg}$ bonds. To ensure the safe operation of the array, an interlock circuit has been designed into the front plane board on the SDSU controller. The interlock is not powered up until the temperature has dropped below $130 \mathrm{~K}$. If the array is accidently operated at higher temperature it can be completely restored with a short bake of a few hours at $50^{\circ} \mathrm{C}$. While the damage done to the SAPHIRA APD can be repaired with a bake out, ideally the initial damage would best be avoided.

There is no specific order to the power-down sequence. The only requirement is that all SPI biases and power signals are brought to ground in unison. The lack of an ordered power-down sequence simplifies the temperature interlock system. The temperature interlock circuit consists of two op-amp comparator circuits. The first monitors the bias voltage across the diode and enables the interlock when the voltage exceeds $0.5 \mathrm{~V}$. If the bias voltage is below $0.5 \mathrm{~V}$, the interlock circuit will allow the detector to be powered up, thus allowing the system to be safely tested at room temperature on the bench.

When the bias voltage exceeds $0.5 \mathrm{~V}$, the override circuit is enabled and the APD can only operate if the temperature is below $130 \mathrm{~K}$. Jumpers on the front plane board allow the temperature inputs for the interlock circuit to be selected from either the temperature sensor built into the SAPHIRA APD or alternatively from the output of the Lakeshore temperature controller.

The output of the comparator circuit drives a series of FETs to power down the APD. The APD is powered down by two mechanisms. The first is triggered when the temperature exceeds $130 \mathrm{~K}$ by triggering the external interrupt on the DSP. The second stage pulls the biases and power to ground independently of the SDSU when the temperature exceeds $135 \mathrm{~K}$.

\section{CRYOSTAT}

The design for the cryostat is based in an existing design for previous instruments built by the RSAA at ANU. The existing cryostat design has been enlarged to accommodate active and passive radiation shields as well as the additional electronics which will be housed within the cryostat.

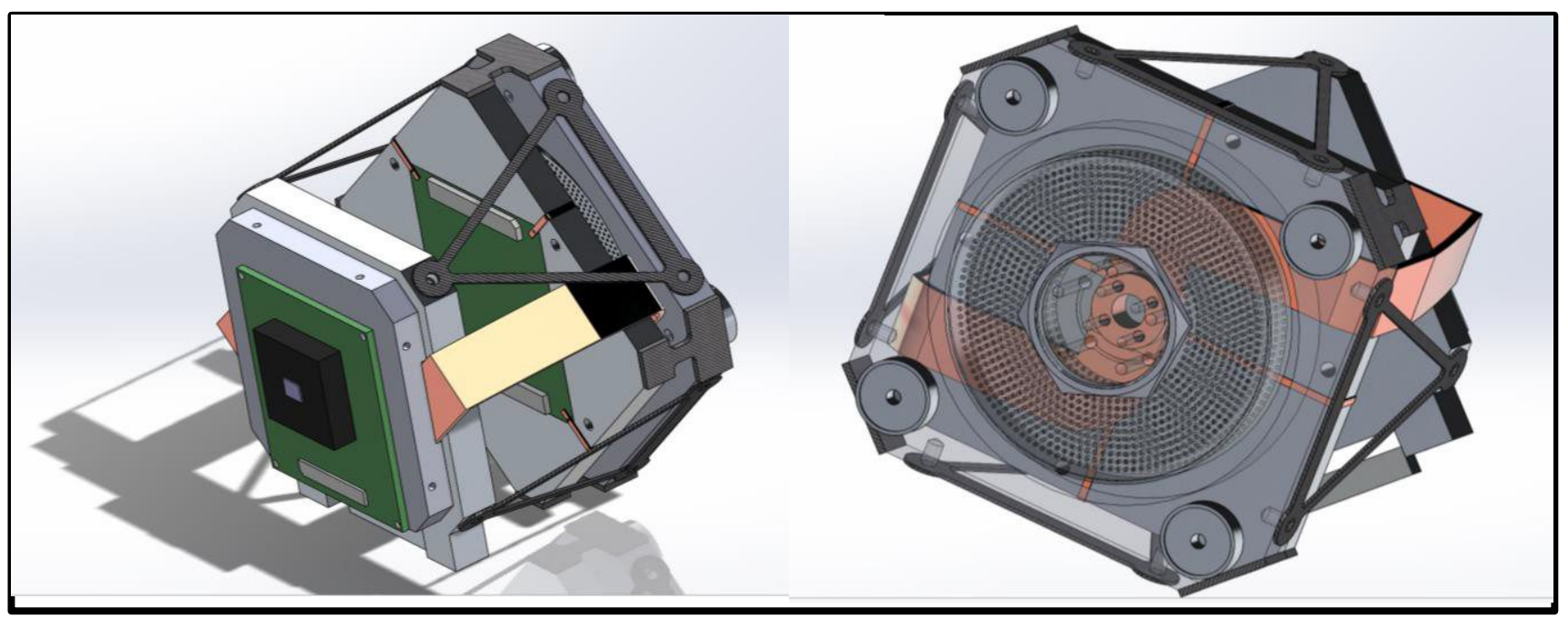

Figure 5. Detector mount and getter assembly. The detector mount is attached to the cryostat via 4 shims which will allow for some optical alignment and adjustment in the focus.

Great care has been taken to ensure that the cryostat that the detector is shielded from radiation sources within the cryostat. The primary heat source within the cryostat is the preamplifier circuit, which has been placed behind the detector and housed within its own cooled enclosure. The location of the preamplifiers behind the detector has complicated the cooling path for the focal plane, requiring the copper straps cooling the focal plane to take a long and circuitous path to the cold head. 
The layout of the cryostat also allows for future expansion of the electronics contained within the cryostat. It is intended that this cryostat will form a testbed for future developments in readout electronics, and so the cryostat requires the capability to accommodate a variety of different designs for the readout chain. Accordingly, the cryostat electronics interface port will be designed to take a range of adapter plates to accommodate a variety of different electrical interfaces including potted feedthroughs and large hermetic circular connectors.

The current design for the cryostat is compact and easily transportable to ensure that the instrument can be shipped and installed at a number of different telescopes around the world. The mechanical attachment interfaces of the cryostat are adaptable to accommodate a variety of installation scenarios and a degree of adjustability has been built into the detector mount to allow for some adjustment in the optical path length.

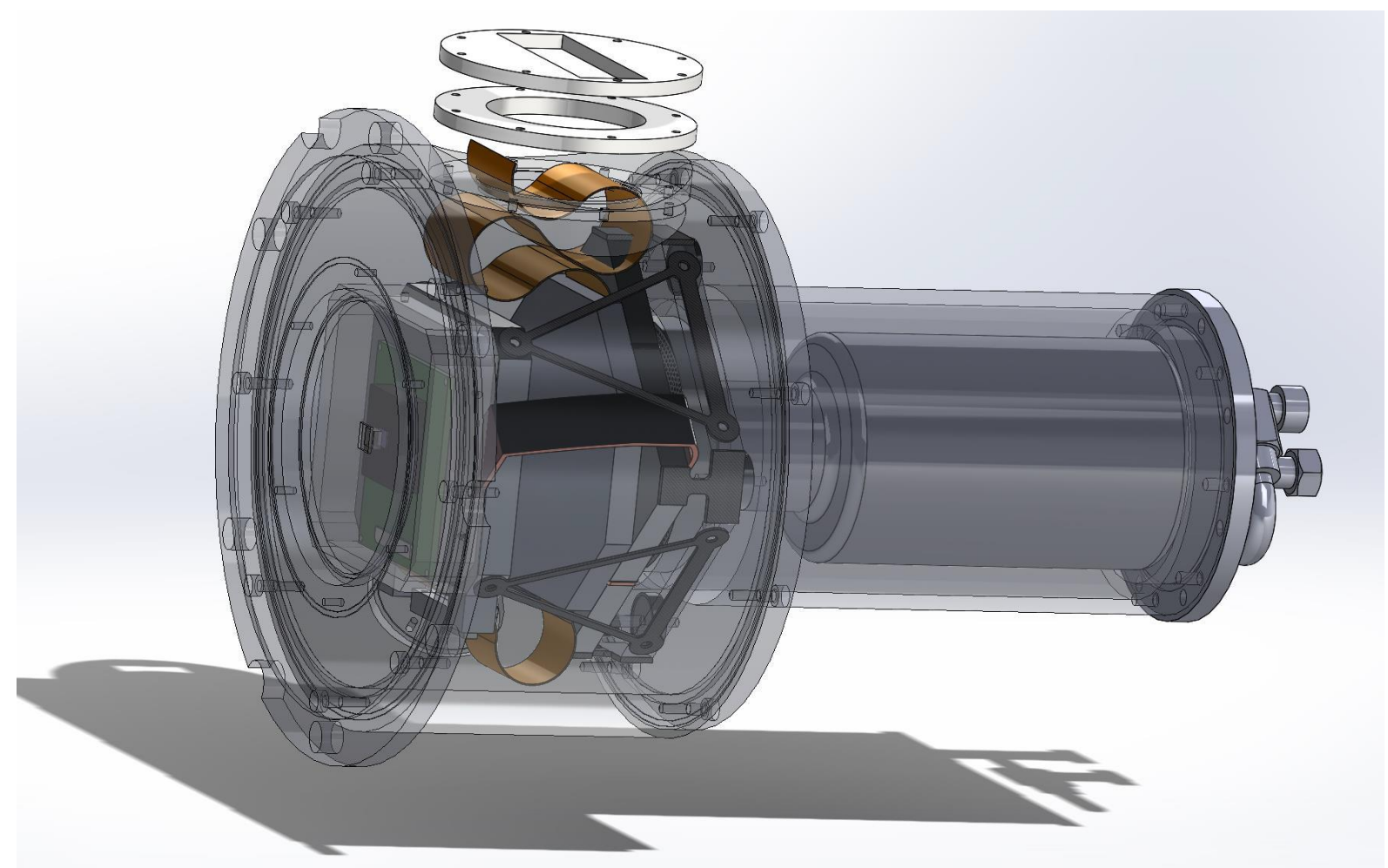

Figure 6. The assembled cryostat. The top of the cryostat shows the two options for adapter plates for the electronics interface; one pate is for a potted flex cable and the other for a large hermetic circular connector.

\section{CONCLUSION}

This Lucky Imager system will form the basis of an invaluable test bed to evaluate and optimise the Selex SAPHIRA APD for the GMT adaptive optics programme while also providing an invaluable tool for gaining expertise and experience with this exciting and revolutionary new technology. When the development of this instrument is complete, it will significantly enhance the capabilities of the ANU $2.3 \mathrm{~m}$ telescope at Siding Spring Observatory.

\section{ACKNOWLEDGEMENTS}

This research was supported under Australian Research Council's Linkage Project funding scheme (LP150100620) in partnership with the Australian National University and Giant Magellan Telescope Organisation. 


\section{REFERENCES}

[1] Atkinson, D., Hall D., Baranec C., Baker I., Jacobsen S. and Riddle R., "Observatory deployment and characterization of SAPHIRA HgCdTe APD Arrays," Proc. SPIE, 9154,12 (2104)

[2] Dopita, M., Hart, J., McGregor, P., Oates, P., Bloxham, G., \& Jones, D., "The Wide Field Spectrograph (WiFeS)", Ap\&SS, 310, 255-268 (2007).

[3] Sharp, R., G. Bloxham, R. Boz, D. Bundy, J. Davies, B. Espeland, Fordman, B., J. Hart, N. Herald, J. Nielsen, A. Vaccarella, C. Vesta, P. Young, P. McGregor, "GMTIFS: The Giant Magellan Telescope integral fields spectrograph and Imager," Proc. SPIE 9908 (2016)

[4] McGregor, P. J., Hart, J., Conroy, P. G., Pfitzner, M. L., Bloxham, G. J., Jones, D. J., Downing, M. D., Dawson, M., Young, P., Jarnyk, M., and Van Harmelen, J., "Gemini near-infrared integral field spectrograph (NIFS)," Proc. SPIE, 4841, 1581-1591, (2003)

[5] McGregor, P. J., Hart, J., Stevanovic, D., Bloxham, G., Jones, D., van Harmelen, J., Griesbach, J., Dawson, M., Young, P., \& Jarnyk, M., "Gemini South Adaptive Optics Imager (GSAOI)," Proc. SPIE, 5492, 1033-1044 (2004)

[6] Goodwin, M., Jenkins, C., Lambert, A., “Adaptive Optics Simulations for Siding Spring,” PASA, 30, 10, (2013)

[7] Goodwin, M., Jenkins, C., Lambert, A., "Characterisation of the Optical Turbulence at Siding Spring," PASA, $30,9,(2013)$ 